\title{
Meningeal Hemangiopericytomas and Meningomas: a Comparative Immunohistochemical and Genetic Study
}

\author{
Saoussen Trabelsi ${ }^{1}$, Nadia Mama ${ }^{2}$, Maroua Chourabi ${ }^{1}$, Maroua Haddaji \\ Mastouri $^{1}$, Mohamed Ladib ${ }^{3}$, Sergey Popov ${ }^{4}$, Anna Burford ${ }^{4}$, Moncef Mokni ${ }^{5}$, \\ Kalthoum Tlili ${ }^{2}$, Hedi Krifa ${ }^{3}$, Chris Jones ${ }^{4}$, Mohamed Tahar Yacoubi ${ }^{5}$, Ali Saad ${ }^{1}$, \\ Dorra H'mida-Ben Brahim ${ }^{1 *}$
}

\begin{abstract}
Background: The meningeal hemangiopericytoma (MHPC) is a vascular tumor arising from pericytes. Most intracranial MHPCs resemble meningiomas (MNGs) in their clinical presentation and histological features and may therefore be misdiagnosed, despite important differences in prognosis. Materials and Methods: We report 8 cases of MHPC and 5 cases of MNG collected from 2007 to 2011 from the Neuro-Surgery and Histopathology departments. All 13 samples were re reviewed by two independent pathologists and investigated by immunohistochemistry (IHC) using mesenchymal, epithelial and neuro-glial markers. Additionally, we screened all tumors for a large panel of chromosomal alterations using multiplex ligation probe amplification (MLPA). Presence of the NAB2-STAT6 fusion gene was inferred by immunohistochemical staining for STAT6. Results: Compared with MNG, MHPCs showed strong VIM (100\% of cases), CD99 (62\%), bcl-2 (87\%), and p16 (75\%) staining but only focal positivity with EMA $(33 \%)$ and NSE (37\%). The p21 antibody was positive in $62 \%$ of MHPC and less than 1\% in all MNGs. MLPA data did not distinguish HPC from MNG, with PTEN loss and ERBB2 gain found in both. By contrast, STAT6 nuclear staining was observed in 3 MHPC cases and was absent from MNG. Conclusions: MNG and MHPC comprise a spectrum of tumors that cannot be easily differentiated based on histopathology. The presence of STAT6 nuclear positivity may however be a useful diagnostic marker.
\end{abstract}

Keywords: Meningeal hemangiopericytoma - immunohistochemistry - NAB2-STAT6 fusion - molecular analysis

Asian Pac J Cancer Prev, 16 (16), 6871-6876

\section{Introduction}

Meningeal hemangiopericytoma (MHPC) is a malignant neoplasm which originates from meningeal capillary pericytes (Stout and Murray, 1942). It has been reported to represent 2 to $4 \%$ of meningeal tumors, comprising less than $1 \%$ of all intracranial tumors(Guthrie et al., 1989). Epidemiological studies reports that MHPC are rare tumors with lower average than meningiomas (MNGs) (Jazayeri et al., 2013) . Most intracranial MHPCs mimic MNGs in their clinical and histopathological presentation, as well as their immunohistochemical profile (Rajaram et al., 2004), and are hence often misdiagnosed.

Meningiomas are neoplasms arising from meningothelial cells of the meninges (Larijani et al., 2014). They constitute approximatively $13-26 \%$ of all intracranial tumours (Ozbayir et al., 2011). They are most often slow-growing benign tumors, however atypical or anaplastic tumors can be found (Ozbayir et al., 2011). Moreover, atypical anaplastic meningioma has diverse radiological manifestations and MRI technology has a certain diagnosis limitations (Wu et al., 2013).

However, MHPC metastasize outside the CNS in $25 \%$ $60 \%$ of cases, whereas meningiomas metastasize only occasionally (Rajaram et al., 2004). Given this important prognostic difference, the correct diagnosis and thus the appropriate treatment strategy should be adopted.

The recent WHO classification describes HPC, solitary fibrous tumours (SFT) and MNG as a new biomarkers has prompted us to look for potential differences in IHC. According to S.Barthelme et al. (2014) presence of the NAB2-STAT6 fusion gene distinguishes SFT from MHPC (Barthelmess et al., 2014). This fusion is not well described in MHPCs and has not been reported in the specific comparison between MNG and MHPC. Investigation of additional molecular markers in this differential diagnosis has also been lacking.

\section{Materials and Methods}

We investigated a cohort of 8 MHPCs and 5 MNGs from adults. MHPC were provided from 3 women and 
5 men; MNG patients were from 3 women and 2 men. Patients' ages ranged from 25 to 81 years. 7 of the 8 MHPC locations were supratentorial: 4 occipital, 2 frontal and 1 temporo-occipital. Only 1 MHPC was located in the cerebellum. MNG tumor location was supra-tentorial in 4 cases and infra-tentorial in 1 case. Clinical symptoms at diagnosis were variable: hemiparesis, disorientation, decreased visual acuity, and headache (Table 1).

\section{Standard histology}

Formalin-fixed paraffin-embedded tissue was sectioned at $4 \mu \mathrm{m}$ and stained with haematoxylin and eosin and reviewed by two pathologists.

\section{Immunohistochemistry}

An immunohistochemical study was performed on formalin-fixed, paraffin-embedded tissue cut at $4 \mu \mathrm{m}$ using polyclonal antibodies (Table 2).

Immunohistochemistry for STAT6 was carried out as a surrogate for the NAB2-STAT6 fusion. Antigen retrieval was performed at $98^{\circ} \mathrm{C}$ with $\mathrm{DAKO}$ antigen retrival solution $\mathrm{pH} 6$ for $20 \mathrm{~min}$. Endogenous peroxidase activity

Table 1. Clinical and histopathological data of patients and their developping tumors

\begin{tabular}{|c|c|c|c|c|c|}
\hline \multirow{2}{*}{ Patients \# } & \multirow{2}{*}{$\begin{array}{c}\text { Age at diagnosis } \\
\text { (year) }\end{array}$} & Sexe & \multicolumn{3}{|c|}{ Tumor } \\
\cline { 3 - 6 } & 70 & M & TP & Mubtype & grade \\
\hline 1 & 17 & F & F & MHPC & II \\
\hline 2 & 61 & M & O & MHPC & II \\
\hline 3 & 34 & F & O & MHPC & III \\
\hline 4 & 46 & M & F & MHPC & I \\
\hline 5 & 34 & F & O & MHPC & III \\
\hline 6 & 52 & M & C & MHPC & I \\
\hline 7 & 46 & F & T & MNG/HPC & III \\
\hline 8 & 50 & M & TO & MNG & III \\
\hline 9 & 81 & M & NA & MNG & II \\
\hline 10 & 25 & F & IE & MNG & II \\
\hline 11 & 65 & F & P & MNG & II \\
\hline 12 & 45 & M & F & MNG & II \\
\hline 13 & & & &
\end{tabular}

*Footnotes: F: female, M: male, C: cerebellum, P: parietal, T: temporal, TP : temporo-parietal, F: frontal; O :Occipital , TO: temporo-occipital, NA: not available, IE: intradural extramedullar, MHPC: meningial hemangiopericytoma, MNG :meningioma

Table 2. Antibodies Results list and Methods in Immunohistochemical Analysis

\begin{tabular}{|c|c|c|c|c|c|}
\hline \multirow{2}{*}{ Antigen } & Antibody and manufacturers & \multirow{2}{*}{ Dilution } & \multirow{2}{*}{ Antigen retrieval } & \multicolumn{2}{|c|}{ Staining (\%) } \\
\cline { 4 - 6 } & & & MHPC & MNG \\
\hline EMA & Dako Clone E29 & $1: 100$ & EDTA PH6 & $60 \%$ & $37 \%$ \\
\hline VIM & Dako Clone Vim 3B4 & $1: 100$ & EDTA PH6 & $100 \%$ & $50 \%$ \\
\hline Bc12 & Dako Clone 124 & $1: 50$ & EDTA PH6 & $87 \%$ & $50 \%$ \\
\hline CD56 & Neomarker Clone 123C3D5 & $1: 50$ & EDTA PH6 & $25 \%$ & $40 \%$ \\
\hline S100 & Dako Clone & $1: 300$ & $/$ & $12 \%$ & $40 \%$ \\
\hline Synaptophysine & Neomarker Clone Rb-SP111-RM & $1: 200$ & EDTA PH6 & $0 \%$ & $0 \%$ \\
\hline P21 & Novocastra Clone 4D10 & $1: 40$ & EDTA PH6 & $62 \%$ & $20 \%$ \\
\hline P16 & Dako Clone K334 & $1: 50$ & EDTA PH6 & $75 \%$ & $40 \%$ \\
\hline CD34 & Dako Clone Qbend 10 & $1: 50$ & EDTA PH6 & $37 \%$ & $40 \%$ \\
\hline NSE & Dako Clone BBS/NC/V1H14 & $1: 50$ & EDTA PH6 & $37 \%$ & $80 \%$ \\
\hline CD99 & Novocastra clone HO36-1-1 & $1: 20$ & EDTA PH9 & $62 \%$ & $40 \%$ \\
\hline ACE & Dako Clone 11-7 & $1: 50$ & EDTA PH6 & $0 \%$ & $0 \%$ \\
\hline F8 & Dako Clone F8/86 & $1: 50$ & EDTA PH6 & $0 \%$ & $0 \%$ \\
\hline Des & Dako clone D33 & $1: 50$ & EDTA PH9 & $0 \%$ & $0 \%$ \\
\hline GFAP & Dako Clone Poly ZCG23 & $1: 300$ & EDTA PH6 & $0 \%$ & $0 \%$ \\
\hline KI67 & Dako Clone MIB1 & $1: 300$ & EDTA PH6 & $27 \%$ & $14 \%$ \\
\hline STAT6 & Santa Cruz ( S-20; sc-621) & $1: 300$ & EDTA PH6 & $37 \%$ & $0 \%$ \\
\hline
\end{tabular}


Meningeal Hemangiopericytomas and Meningomas - a Comparative Immunohistochemical and Genetic Study.

was blocked with $3 \%$ hydrogen peroxide in methanol.

The detection system used was Novolink Polymer (Leica Microsystems, Newcastle Ltd) with diaminobenzidine as a chromogen. The slides were counterstained with hematoxylin.

\section{DNA extraction}

Tumor genomic DNA was extracted from either fresh tissue using a standard phenol:chloroform protocol, or FFPE tissues according to The QIAamp DNA FFPE Tissue kit according to the manufacturers protocol (QIAGEN).

\section{Multiplex ligation probe amplification (MLPA)}

MLPA is a multiplex PCR technique in which up to 45 specific sequences are simultaneously quantified in a single reaction. For this multigenic technique, a specifically designed set of probes was used to test for chromosomal abnormalities - SALSA MLPA Kits
P105, P370 and P088 (MRC Holland, Amsterdam, The Netherlands).

Fragments were separated by electrophoresis on the ABI Prism 310 capillary genetic analyser. Data analysis was performed with "GeneMarker ${ }^{\mathbb{B}}$ " software (SoftGenetics). Thresholds to detect losses and gains of genetic material were set respectively at 0.75 and 1.25.

\section{Results}

\section{Histology}

Microscopically, MHPCs showed a variable cell proliferation rate and focal necrosis. All studied MNG showed a high cellular proliferation rate and are thus classified as a high grade MNG. The MHPC group showed oval and atypical cells with high vascularity. Areas presenting surrounding branched vessels exhibiting a staghorn pattern were specifically seen in MHPCs.

Table 3. Genetic and immunohistochemistry results

\begin{tabular}{|c|c|c|c|c|c|c|c|c|c|}
\hline \multirow{3}{*}{\multicolumn{2}{|c|}{ Tumor \# }} & \multicolumn{8}{|c|}{ MLPA } \\
\hline & & & & & CDKN2A & PTEN & EGFR & TP53 & ERBB2 \\
\hline & & $1 \mathrm{p} 36$ & $19 \mathrm{q} 13$ & $7 \mathrm{q} 36$ & 9 p21 & $10 \mathrm{q} 26$ & $11 \mathrm{p} 11$ & $17 q$ & \\
\hline \multirow{8}{*}{ MHPC } & $\# 1$ & NS & gain & NS & NS & Loss & NS & NS & NS \\
\hline & $\# 2$ & gain & NS & NS & NS & gain & gain & NS & NS \\
\hline & $\# 3$ & gain & gain & Loss & NS & NS & NS & NS & NS \\
\hline & $\# 4 *$ & gain & gain & Loss & NS & Loss & NS & NS & NS \\
\hline & $\# 5$ & gain & gain & gain & NS & Loss & NS & NS & NS \\
\hline & \#6 & gain & NS & Loss & NS & NS & NS & NS & NS \\
\hline & $\# 7$ & gain & NS & NS & NS & NS & NS & Loss & NS \\
\hline & $\# 8^{*}$ & gain & gain & NS & NS & Loss & gain & NS & gain \\
\hline \multirow{5}{*}{ MNG } & $\# 9$ & NS & NS & NS & NS & Loss & NS & NS & NS \\
\hline & $\# 10$ & gain & gain & NS & gain & Loss & gain & gain & gain \\
\hline & $\# 11^{*}$ & NS & NS & NS & NS & Loss & NS & NS & gain \\
\hline & $\# 12$ & NS & gain & NS & loss & Loss & NS & NS & NS \\
\hline & $\# 13$ & gain & NS & NS & loss & Loss & gain & NS & NS \\
\hline \multirow{3}{*}{\multicolumn{2}{|c|}{ Tumor \# }} & \multicolumn{8}{|c|}{ IHC } \\
\hline & & \multicolumn{8}{|c|}{ Antigen } \\
\hline & & VIM & Syn & ACE & F8 & Des & GFAP & EMA & CD34 \\
\hline \multirow{8}{*}{ MHPC } & $\# 1$ & +++ & - & - & - & - & - & - & + \\
\hline & $\# 2$ & + & - & - & - & - & - & - & - \\
\hline & $\# 3$ & + & - & - & - & - & - & + & + \\
\hline & $\# 4 *$ & + & - & - & - & - & - & $+f$ & - \\
\hline & $\# 5$ & + & - & - & - & - & - & - & - \\
\hline & $\# 6$ & ++ & - & - & - & - & - & $+f$ & ++ \\
\hline & $\# 7$ & +++ & - & - & - & - & - & +++ & - \\
\hline & $\# 8^{*}$ & +++ & - & - & - & - & - & ND & ++ \\
\hline \multirow{5}{*}{ MNG } & $\# 9$ & + & - & - & - & - & ND & + & ++ \\
\hline & $\# 10$ & $+f$ & - & - & - & - & - & - & - \\
\hline & $\# 11 *$ & + & - & - & - & - & - & ++ & - \\
\hline & $\# 12$ & ND & - & - & - & - & - & + & - \\
\hline & $\# 13$ & + & - & - & - & - & - & $+f$ & $+f$ \\
\hline
\end{tabular}




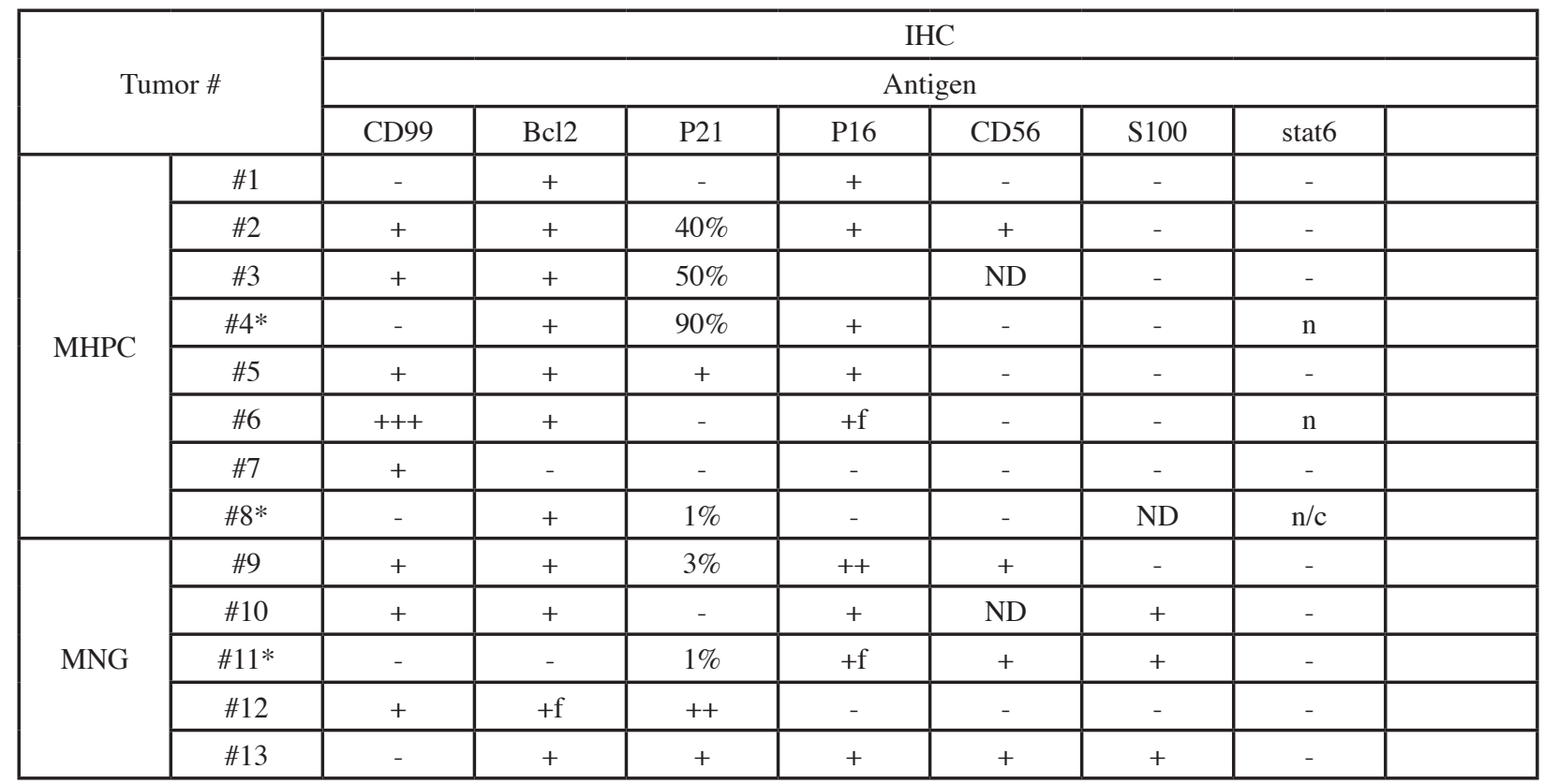

Footnotes: MHPC: Meningial Hemaniopericytoma, MNG: meningioma, (NS): normal status, (-) : negative staining ,(+): weak positive staining, (++): moderate positive staining, $(+++)$ : high positive staining, (+f) : focal staining, $(\mathrm{n})$ : nuclear staining, $(\mathrm{c})$ : cytoplasmic staining, $(\mathrm{n} / \mathrm{c})$ : nuclear and cytoplasmic staining, ND : not determined. $\left(^{*}\right)$ : Tumor section with STAT6 staining presented in figure 1

Table 4. Hemangiopericytomas and Meningiomas histological description

\begin{tabular}{|c|c|c|c|c|c|c|c|c|}
\hline \multirow{2}{*}{ Tumor \# } & \multirow{2}{*}{ Proliferation } & \multirow{2}{*}{ Cell shape } & Vascularity & \multirow{2}{*}{ Collagen } & \multirow{2}{*}{ Mitosis } & \multirow{2}{*}{ Necrosis } & \multicolumn{3}{|c|}{ Tumor } \\
\cline { 5 - 9 } & & & & & & & Subtype & Grade \\
\hline 1 & $\mathrm{H}$ & $\mathrm{O}, \mathrm{Fu}$ & $\mathrm{H}$ & + & Rare & - & MHPC & II \\
\hline 2 & $\mathrm{Var}$ & $\mathrm{O}, \mathrm{R}$ & $\mathrm{H}$ & - & Frq & - & MHPC & II \\
\hline 3 & $\mathrm{H}$ & $\mathrm{O}, \mathrm{Fu}$ & $\mathrm{H}$ & - & Rare & + & MHPC & III \\
\hline 4 & $\mathrm{H}$ & $\mathrm{O}$ & $\mathrm{M}$ & + & Rare & + & MHPC & III \\
\hline 5 & $\mathrm{H}$ & $\mathrm{O}, \mathrm{Fu}$ & $\mathrm{H}$ & + & Rare & + & MHPC & II \\
\hline 6 & $\mathrm{H}$ & $\mathrm{O}$ & $\mathrm{H}$ & + & Rare & - & MHPC & III \\
\hline 7 & $\mathrm{H}$ & $\mathrm{O}, \mathrm{R}$ & $/$ & - & Frq & - & MHPC & II \\
\hline 8 & $\mathrm{M}$ & $\mathrm{Fu}$ & $\mathrm{H}$ & - & - & + & HPC,MNG & III \\
\hline 9 & $\mathrm{H}$ & $\mathrm{O}$ & $/$ & - & Frq & + & MNG & III \\
\hline 11 & $\mathrm{M}$ & $\mathrm{O}$ & $\mathrm{M}$ & - & Rare & - & MNG & II \\
\hline 10 & $\mathrm{H}$ & $\mathrm{Fu}, \mathrm{R}$ & $/$ & - & Frq & - & MNG & II \\
\hline 12 & $\mathrm{H}$ & $\mathrm{R}$ & $/$ & - & - & - & MNG & II \\
\hline 13 & $\mathrm{H}$ & $\mathrm{Fu}$ & $\mathrm{H}$ & - & Frq & - & MNG & II \\
\hline
\end{tabular}

Footnotes: (H):high, (M): moderate, (/) : nothing to note, (Var): variable, $(\mathrm{O})$ : oval, (Fu) : fusiform, $(\mathrm{R})$ : rounded, (-) : absent, (+): present, (frq) : frequent, (MHPC): meningial hemaniopericytoma, (MNG): meningioma

Mitoses were frequently observed in MNG but were rare in MHPC (Figure 1, suplementary Table).

\section{Immunohistochemistry}

No immunoreactivity was seen for ACE, F-VIII, Desmin, Synaptophysin and GFAP in any analysed samples. MHPCs express the VIM, CD34, CD99 and Bcl2 $(\# 8,4,5,7$ cases ) in the neoplastic cells more than MNGs $(\# 2,1,3,2)$. EMA expressing rates were higher in MNGs (\#3) than MHPCs (\#2) (Figure 1).p21 was positive in 62\% of MHPCs and less than $1 \%$ in all MNGs.

Based on morphological and immunohistochemical staining features, a final diagnosis of anaplasic meningeal hemangiopericytoma was made in all 8 studied tumors.

\section{STAT6 expression}

As expected, the 5 MNGs were negative for STAT6 expression, used a surrogate for the NBA2-STAT6 fusion gene. Nuclear staining was exclusively present on 3 out of 8 MHPC specimens. 2 positive MHPCs specimens represent a first tumor and its relapse (tumor \#4 and \#6). The third positive MHPC for STAT6 was according to histology and IHC investigations set as an uncertain diagnosis case. The STAT6 labelling showed a focal area of nuclear positivity in addition to abundant cytoplasmic staining in the same tumor section (tumor \# 8) (Figure 1).

\section{Molecular analysis}

We used a molecular approach based on copy 


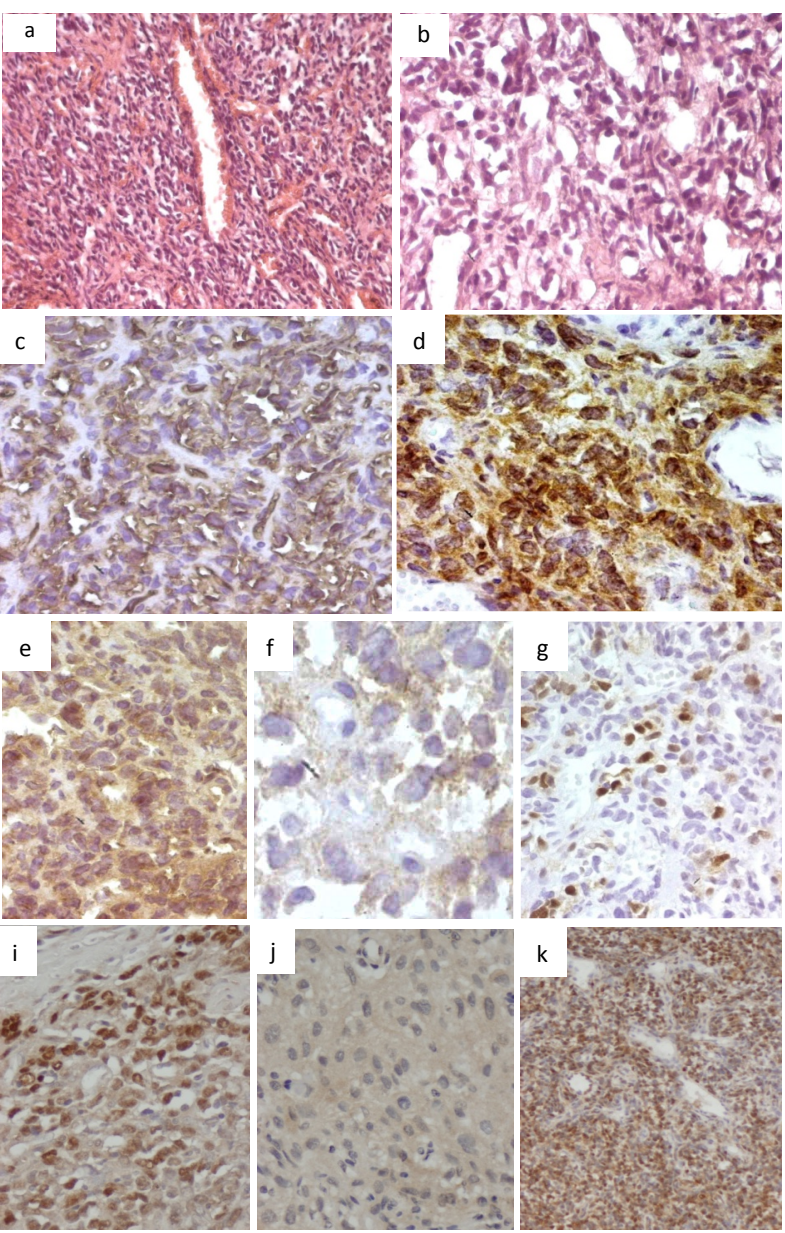

Figure 1. Histopathology of MHPC. a) HE staining $\mathrm{x}$ 200: proliferation of globular cells surrounding branched vessels exhibiting straghorn pattern; b) HE staining x 400: the tumoral cells show atypia and nuclear hyperchromasia; c) AntiCD34 intense positivity of the tumor cells; d) Anti-bcl2 intense positivity of the tumor cells; e) Anti-CD99 mild positivity of the tumor cells; f) Anti-EMA weak positivity of the tumor cells; g) Anti-P16 nuclear positivity of the tumor cells; i) Anti STAT6 nuclear positivity of the HPC tumor cells (tumor \# 4); j) Anti STAT6 negative stainig of the MNG tumor cells (tumor \# 11); k) Anti STAT6 nuclear and cytoplasmic staining of the tumor cells (tumor \# 8)

number analysis to investigate both MHPC and MNG. An overview of molecular results is included in Table 3.

Both MHPC and MNG presented alterations of $1 \mathrm{p} 36$ and ERBB2 regions, and had normal statuses of 3p, 7q3, 19q13. CDKN2A loss was exclusively detected on MNG samples. Of note, PTEN loss was present in both MHPCs (4 MHPC out of 8) and MNGs (3 MNG out of 5).

\section{Discussion}

Intracranial MHPC is a dural-based neoplasm that highly mimics MNGs. The World Health Organization has separated MHPCs from MNGs since 1993, and individualized MHPCs as a "mesenchymal, nonmeningothelial" tumor (Chan et al., 2012). With the exception of MHPC clinical outcome, there are few distinguishing features between MHPC and MNG.

Chiechi et al reported that MHPC occurs in locations similar to MNG (Chiechi et al., 1996; Akiyama et al.,
2004). They are found mostly at supratentorial, less frequently at infratentorial, very rarely intraventricular and even intraparenchymal locations have been reported (Muttaqin et al., 1991; Abrahams et al., 1999; Alen et al., 2001; Kuzeyli et al., 2003). Similarly, most of MHPC as well as MNG in our cases are in a supratentorial location.

The pathological features crucial for the diagnosis also remain the most controversial. Further, the immunoprofiles of MHPC and MNG were reported as slightly different (Shoji et al., 2002). The most important IHC markers thought to be diagnostically useful are EMA and CD34. The EMA is an epithelial marker reported positive in MNG but focal staining occurring in some MHPCs led to diagnostic uncertainty (Rajaram et al., 2004). CD34 is thought to be specific for MHPCs but was also reported to be controversial with a positive staining noted in MNG (Rajaram et al., 2004). However, Bcl-2 has been reported as very sensitive marker to MHPCs (Rajaram et al., 2004).

In our study, EMA as a highly sensitive meningothelial marker was found to be positive in $60 \%$ of our MNGs. Nonetheless, an EMA focal staining was observed in $33 \%$ of MHPCs. Thus EMA expression cannot exclude the diagnosis of MHPC. Bcl-2 was positive in $62 \%$ of MHPCs, however a focal weak signal was also observed in $40 \%$ of MNG. As reported by other studies CD99 proves to be another marker that MHPC strongly express (Rajaram et al., 2004). In our series it proved to be a sensitive marker with MHPC (62\%) but still remains positive in MNG (40\%). In fact, both CD99 and Bcl-2 highlight the extensive overlap between the two tumor groups.

In accordance with Shoji et al, the majority of MHPCs strongly express p21, whilst it is almost entirely absent from MNGs $\mathrm{p} 21$, is the product of the WAF1/CIP1/SDI gene and an inhibitor of cyclin-dependent kinases. It has been clearly demonstrated that prognostic significance of p21 is enhanced in combination with other apoptotic factors (i.e. p53) and associated with a better outcome (Shoji et al., 2002). The absence of p21 expression in MNG may be associated with their high grade.

We noted that the genetic profiles of MHPC and MNG were very similar except for CDKN2A loss predominantly present in MNGs. CDKN2A loss was previously described in anaplasic tumors and it matched with the high-grade histological description in our MNG samples (Bostrom et al., 2001).

ERBB2 belongs to the receptor tyrosine kinase family I. It has an important role in regulating the anti-apoptotic phosphatidyl inositol 3-kinase (PI3K)/protein kinase B (PKB)/AKT signalling pathway (Gilbertson et al., 1998; Elenius et al., 1999). ERBB2 is located on chromosome 17q11.2-q12, a region frequently gained in several tumors as found in our MNGs (de Bont et al., 2008).

PTEN is located at 10q23.3, a chromosomal region frequently implicated in tumor malignancy. In contrast to a previous report (Peters et al., 1998), we found PTEN loss in 3 MNG. This gene loss was also detected in 4 MHPCs and is therefore not specific to either entity.

In our study, both IHC markers and genetic profiles did not discriminate MNG from MHPC. A fusion gene between NAB2 and STAT6 has been recently identified as potential marker specific for SFT (Barthelmess et al., 
2014).

Based on Schweizer et al study, we use IHC staining with the STAT6 antibody that recognizes C-terminal portion of STAT6. STAT6 protein mainly localizes to in the cytoplasm whereas the NAB2 protein localizes to in the nucleus. In case of fusion between NAB2 and STAT6, the fusion protein preserves the C-terminal portion of STAT6 connected with the 3' portions of the NAB2 (UniProtKB $\mathrm{Q} 15742)$. For this reason, if the fusion occurs we expect to find the STAT6 staining in the nucleus(Schweizer et al., 2013).

MHPC sections presented STAT6 nuclear staining in 3 samples, whereas MNG were all negative, adding more support to previous studies (Iwaki et al., 1988) that reported the presence of NAB2-STAT6 fusion gene more frequently in MHPCs then MNGs. This molecular difference could be attributed to distinctive tumor steam cell origin and/or tumorogenesis pathways. Our conclusions need to be followed up by an enlargement of our sample cohort.

Interestingly, a case with highly uncertain diagnosis presented high cell proliferation. According to the immunoprofile with $\mathrm{CD} 34$ and $\mathrm{Bcl} 2$ positivity, it is more likely consistent with MHPC but still uncertain. The STAT6 IHC results showed a focal area of nuclear staining in addition to abundant cytoplasmic staining in the same tumor section. This could provide a furthermore argument to propose the MHPC as a final diagnosis and show the utility of STAT6 in the differential diagnoses of such lesions.

\section{Acknowledgements}

We are grateful to all patients for their contribution. We also extend special thanks to all members of GETUC “ 'Groupe d'Etude des Tumeurs Cerebrales" and Glioma team at the Divisions of Molecular Pathology and Cancer Therapeutics at the Institute of Cancer Research in Sutton. UK.

\section{References}

Abrahams JM, Forman MS, Lavi E, et al (1999). Hemangiopericytoma of the third ventricle. Case report. $J$ Neurosurg, 90, 359-62.

Akiyama M, Sakai H, Onoue H, et al (2004). Imaging intracranial haemangiopericytomas: study of seven cases. Neuroradiol, 46, 194-7.

Alen JF, Lobato RD, Gomez PA, et al (2001). Intracranial hemangiopericytoma: study of 12 cases. Acta Neurochir (Wien), 143, 575-86.

Barthelmess S, Geddert H, Boltze C, et al (2014). Solitary fibrous tumors/hemangiopericytomas with different variants of the NAB2-STAT6 gene fusion are characterized by specific histomorphology and distinct clinicopathological features. Am J Pathol, 184, 1209-18.

Bostrom J, Meyer-Puttlitz B, Wolter M, et al (2001). Alterations of the tumor suppressor genes CDKN2A (p16(INK4a)), p14(ARF), CDKN2B (p15(INK4b)), and CDKN2C (p18(INK4c)) in atypical and anaplastic meningiomas. $\mathrm{Am}$ J Pathol, 159, 661-9.

Chan JK, Cheuk W, Ho LC, et al (2012). Recurrent meningeal hemangiopericytoma with multiple metastasis and hypoglycemia: a case report. Case Rep Med, 2012, 628756. Chiechi MV, Smirniotopoulos JG, Mena H (1996). Intracranial hemangiopericytomas: MR and CT features. AJNR Am J Neuroradiol, 17, 1365-71.

de Bont JM, Packer RJ, Michiels EM, et al (2008). Biological background of pediatric medulloblastoma and ependymoma: a review from a translational research perspective. Neuro Oncol, 10, 1040-60.

Elenius K, Choi CJ, Paul S, et al (1999). Characterization of a naturally occurring ErbB4 isoform that does not bind or activate phosphatidyl inositol 3-kinase. Oncogene, 18, 2607-15.

Gilbertson RJ, Clifford SC, MacMeekin W, et al (1998). Expression of the ErbB-neuregulin signaling network during human cerebellar development: implications for the biology of medulloblastoma. Cancer Res, 58, 3932-41.

Guthrie BL, Ebersold MJ, Scheithauer BW, et al (1989). Meningeal hemangiopericytoma: histopathological features, treatment, and long-term follow-up of 44 cases. Neurosurg, 25, 514-22.

Iwaki T,Fukui M, Takeshita I, etal(1988). Hemangiopericytoma of the meninges: a clinicopathologic and immunohistochemical study. Clin Neuropathol, 7, 93-9.

Jazayeri SB, Rahimi-Movaghar V, Shokraneh F, et al (2013). Epidemiology of primary CNS tumors in Iran: a systematic review. Asian Pac J Cancer Prev, 14, 3979-85.

Kuzeyli K, Cakir E, Karaarslan G, et al (2003). Primary parenchymal cerebral cystic haemangiopericytoma: a 5 -year follow up of disease progression. Australas Radiol, 47, 88-91.

Larijani L, Madjd Z, Samadikuchaksaraei A, et al (2014). Methylation of O6-methyl guanine methyltransferase gene promoter in meningiomas--comparison between tumor grades I, II, and III. Asian Pac J Cancer Prev, 15, 33-8.

Muttaqin Z, Uozumi T, Kuwabara S, et al (1991). Intraventricular hemangiopericytoma--case report. Neurol Med Chir (Tokyo), 31, 662-5.

Ozbayir T, Malak AT, Bektas M, et al (2011). Information needs of patients with meningiomas. Asian Pac J Cancer Prev, 12, 439-41.

Peters N, Wellenreuther R, Rollbrocker B, et al (1998). Analysis of the PTEN gene in human meningiomas. Neuropathol Appl Neurobiol, 24, 3-8.

Rajaram V,BratDJ,Perry A(2004). Anaplastic meningioma versus meningeal hemangiopericytoma: immunohistochemical and genetic markers. Hum Pathol, 35, 1413-8.

Schweizer L, Koelsche C, Sahm F, et al (2013). Meningeal hemangiopericytoma and solitary fibrous tumors carry the NAB2-STAT6 fusion and can be diagnosed by nuclear expression of STAT6 protein. Acta Neuropathol, 125, 651-8.

Shoji T, Tanaka F, Takata T, et al (2002). Clinical significance of p21 expression in non-small-cell lung cancer. J Clin Oncol, 20, 3865-71.

Stout AP, Murray MR (1942). Hemangiopericytoma: a vascular tumor featuring zimmermann's pericytes. Ann Surg, 116, 26-33.

Wu QW, Yan RF, Li Q, et al (2013). Magnetic resonance image manifestations of the atypical meningioma. Asian Pac J Cancer Prev, 14, 6337-40. 